\title{
The association between the expression of PAR2 and TMEM16A and neuropathic pain
}

\author{
MENG ZHANG ${ }^{1 *}$, CUN-XIANG GAO ${ }^{2 *}$, YAN-PING WANG ${ }^{3}$, KE-TAO MA ${ }^{4}, \mathrm{LI} \mathrm{LI}^{4}$, \\ JIANG-WEN YIN ${ }^{1}$, ZHI-GANG DAI ${ }^{1}$, SHENG WANG ${ }^{1}$ and JUN-QIANG SI ${ }^{4,5}$ \\ Departments of ${ }^{1}$ Anesthesiology and ${ }^{2}$ Urology, The First Affiliated Hospital of Shihezi University, \\ Shihezi, Xinjiang 832002; ${ }^{3}$ Houbo College, Xinjiang Medical University, Karamay, Xinjiang 834000; \\ ${ }^{4}$ Electrophysiological Laboratory, Laboratory of Xinjiang Endemic and Ethnic Diseases; \\ ${ }^{5}$ Department of Physiology, Medical College, Shihezi University, Shihezi, Xinjiang 832002, P.R. China
}

Received September 15, 2017; Accepted December 8, 2017

DOI: $10.3892 / \mathrm{mmr} .2017 .8295$

\begin{abstract}
Chronic constriction injury (CCI) of the sciatic nerve may induce dorsal root ganglion (DRG) neuronal hyperexcitability and behaviorally expressed hyperalgesia. CCI is a model of neuropathic pain. To investigate the association between the expression of protease activated receptor 2 (PAR2), TMEM16A and neuropathic pain, the expression of PAR2 and TMEM16A proteins in the DRG neurons of rats following CCI of the sciatic nerve was investigated. Following the creation of the CCI model, the thermal withdrawal latency (TWL) was examined by a hot plate test. An immunofluorescence assay and western blot assay were performed to determine the expression of PAR2 and TMEM16A proteins in the ipsilateral $\mathrm{L}_{4-6}$ DRG neurons. The concentration of inositol 1,4,5-triphosphate $\left(\mathrm{IP}_{3}\right)$ in the $\mathrm{L}_{4-6}$ DRG was determined by ELISA. In the CCI-D7 (7 days after CCI) and CCI-D14 (14 days after CCI) treatment groups, the TWL of rats was significantly shorter than that in the sham operated group $(\mathrm{P}<0.01 ; \mathrm{n}=12)$. The expression of PAR2 and TMEM16A proteins in the CCI-D7 and CCI-D14 groups were significantly upregulated compared with the sham operated group $(\mathrm{P}<0.05 ; \mathrm{n}=12)$. Additionally, it was revealed that PAR2 and TMEM16A were co-expressed in DRG neurons. It was also observed that $\mathrm{IP}_{3}$ significantly increased in the CCI-D7 and CCI-D14 groups compared with the sham operation group $(\mathrm{P}<0.05 ; \mathrm{n}=6)$ as PAR2 and TMEM16A also increased. These findings suggest that the upregulation of PAR2 and TMEM16A in DRG neurons,
\end{abstract}

Correspondence to: Dr Jun-Qiang Si, Department of Physiology, Medical College, Shihezi University, 192 Beier Road, Shihezi, Xinjiang 832002, P.R. China

E-mail: sijunqiang@shzu.edu.cn

${ }^{*}$ Contributed equally

Key words: neuropathic pain, protease activated receptor 2, TMEM16A, dorsal root ganglia, thermal hyperalgesia the co-expression of the two proteins and increasing $\mathrm{IP}_{3}$ are critical to the development of neuropathic pain.

\section{Introduction}

Neuropathic pain refers to lesions of the somatosensory system or pain caused by the disease (1). According to the survey, the incidence of neuropathic pain is different, the British about $1 \%$ (2), about $1.5 \%$ in the United States (3), Canada $17.9 \%$ (4), in China, the incidence rate is about $7 \%$. More than $2 / 3$ of patients can not effectively relieve pain, which not only seriously affects the quality of life of patients (5), but also increased the burden on society (6). At present, we mainly use analgesic drugs to treat neuropathic pain. Unfortunately, pharmacological treatment of neuropathic pain is more symptomatic, because the mechanisms responsible for the pain remain unclear. Therefore, a better understanding of the mechanism of neuropathic pain is important for the treatment of the disease. Peripheral nerve injury-induced neuropathic pain have been related to increased excitability occurring in peripheral and central nervous systems as the product of enhanced synaptic excitation, decreased synaptic inhibition, and increased neuronal responsiveness. Pain of first with the participation and regulation is the peripheral nervous system, including the DRG neurons. Pain is initiated with the detection of noxious stimuli at terminals of DRG neurons innervated in peripheral nervous system.

PAR2 is highly expressed in the peripheral and central nervous system and belongs to the $\mathrm{G}$ protein coupled receptors (GPCR) (7). Recent research shows that PAR2 can promote neurogenic inflammation and pain by stimulating sensory neurons (8), the activation of PAR2 increased the release of $\mathrm{IP}_{3}$, further increased the intracellular concentration of calcium ion (9), the latter leads to calcium activated chloride channels (CaCCs) opening. In the event of pain or inflammation, $\mathrm{CaCCs}$ can also increase the excitability of DRG neurons, and then aggravate the occurrence of neuropathic pain mediated by tissue injury $(10,11)$.

In 2008, researchers found TMEM16A is the molecular basis for CaCCs (12). It is a kind of heat sensitive protein, which has been found to be involved in many kinds of 
physiological functions, hyperalgesia and pain can also be significantly reduced in TMEM16A knockout mice (13). In addition, the activation of PAR2 increased the release of $\mathrm{IP}_{3}$, further increased the intracellular concentration of calcium ion (9). With the increase of intracellular calcium in concentration, the activation threshold of TMEM16A was decreased and the activating current of TMEM16A was enhanced (14). These results suggest that TMEM16A may participate in neuropathic pain.

We have shown that TMEM16A were widely expressed in DRG neurons (15), however, we do not know whether PAR2 were expressed in DRG neurons, whether the PAR2 and TMEM16A are co-expression in DRG neurons, whether they play the role in the molecular mechanism of neuropathic pain is still unknown. Therefore, in this experiment, we suppose increasing expression of PAR2 increased the release of $\mathrm{IP}_{3}$, further increased the intracellular concentration of calcium ion, the activation threshold of TMEM16A was decreased and the expression of TMEM16A was increased. We observe the expression of PAR2 and TMEM16A in DRG neurons and its changes with time in neuropathic pain, then we detected the concentration of $\mathrm{IP}_{3}$ to explore the relationship of PAR2 and TMEM16A in the development of neuropathic pain.

\section{Materials and methods}

Animals. A total of 114 male Spraque-Dawley (SD) rats, weighing 200-220 g, 8-12 weeks, provided by the Experimental Animal Center of Xinjiang Medical University, Urumqi, China (certificate no. SCXK 2003-0001), lived under standard laboratory conditions for $12 \mathrm{~h}$ light and $12 \mathrm{~h}$ darkness. Room temperature $\left(20-22^{\circ} \mathrm{C}\right)$, humidity $(50-60 \%)$ and provide clean food and water. The present study was conducted in accordance with approval from the Institutional Ethics Review Board (IERB) at the First Affiliated Hospital of the Shihezi University School of Medicine (IERB no. SHZ2010LL01). All experiments were carried out according to the Ethical Guidelines for Investigations of Experimental of International Association for the Study of Pain. All experimental procedures minimized the suffering and number of rats used, within the premises of achieving the experimental purpose (16).

Experimental grouping and animal modeling. Male SD rats were randomly divided into 3 groups, sham group, CCI-D7 group and CCI-D14 group. In short, SD rats were anesthetized with $10 \%$ chloral hydrate $(0.3 \mathrm{~g} / \mathrm{kg})$ by intraperitoneal injection and fixed on the operating table in prone position. For skin preparation and disinfection of rats, open the left hind leg skin with a knife, strip muscles and tissues with forceps, the sciatic nerve of the left hind paw was exposed using blunt dissection through the biceps femoris muscle at the midthigh, and then mid-sciatic nerve was loosely tied using 4 ligatures (chromic catgut, 4-0; Ethicon, Inc., Cincinnati, OH, USA) proximal to the sciatic trifurcation at $1 \mathrm{~mm}$ spacing, which can induce mild twitching leg strength. The rats in the sham group only exposed the sciatic nerve without ligation. The wound after operation was sutured using surgical thread (Mersilk, 5.0; Ethicon, Inc.) and operation sterilization was performed using iodophor solution and triple antibiotic ointment (Tricin; Merck KGaA, Darmstadt, Germany). After surgery, the rats were housed in separate cages and monitor recovery period. There were significant differences in thermal withdrawal latency (TWL) between the contralateral side of CCI group and sham group, and TWL of ipsilateral side in CCI group were declined by at least $30 \%$ in contrast with sham group, indicating CCI model was successfully established. Thermal latencies of ipsilateral and contralateral paw withdrawal were assessed before surgery (one day, baseline) and on days 1, 3, 5, 7, 10 and 14 after surgery in sham group and CCI group.

Hot-plate testing. CCI induced hyperalgesia was assessed by the hind paw thermal withdrawal test using a hot-plate instrument (Hot Plate; Ugo Basile Biological Research Apparatus, Ugo Basile, Italy). A total of 24 rats were used to have the Hot-plate testing in the result, 12 in the Sham group and 12 in the CCI group. Each rat was placed in the plastic box on a hot plate at $58^{\circ} \mathrm{C}$ and allowed to habituated for $30 \mathrm{~min}$ before testing. The duration from onset of application of thermal stimulus to either hind-paw lick/jump/withdraw was defined as withdrawal latency (TWL). In order to avoid the damage of tissue, cut-off time for paw withdrawal latency was maintained at $30 \mathrm{sec}$ in all cases. The TWL of each rat at each time point was recorded in sec and was repeated three times with 4 min interval. The TWL was obtained by calculating the mean of three withdrawal latencies. All the subjects were tested in the morning from 8:00am to 10:00am. Compared with the preoperative, TWL decreased more than $30 \%$ as a model of success criteria (17).

DRG harvesting. Rats of all groups were anesthetized with $10 \%$ chloral hydrate $(0.3 \mathrm{~g} / \mathrm{kg})$ by intraperitoneal injection. The skin overlying the lumbar region was cut away. A laminectomy of the lumbar region was then carried out. After exposing sciatic nerve, attached ipsilateral lumbar DRGs $\left(\mathrm{L}_{4-6}\right)$ were carefully dissected and rapidly transferred into liquid nitrogen and kept at $-80^{\circ} \mathrm{C}$. Ipsilateral L4-6 DRGs were collected from animals on days 7 and 14 following CCI, when neuropathic pain was developed. The $\mathrm{L}_{4-6}$ DRGs of sham group were harvested after two days of surgery.

Immunofluorescence. Immunofluorescent staining of PAR2 and TMEM16A in DRGs were performed according to Miao et al (18) with some modification 36 rats were randomly divided into 3 groups. Rats from sham group $(n=12)$ and days $7(n=12)$ and $14(n=12)$ post-CCI were anaesthetized with $10 \%$ chloral hydrate $(0.3 \mathrm{~g} / \mathrm{kg})$, and then perfused through the aorta with $0.9 \%$ normal saline, followed by fresh $4 \%$ paraformaldehyde in PBS for 10 min for tissue fixation. The $\mathrm{L}_{4-6} \mathrm{DRGs}$ were removed rapidly and placed in $4 \%$ paraformaldehyde in PBS for $24 \mathrm{~h}$. Then the $\mathrm{L}_{4-6}$ DRGs were sectioned (thickness, $5 \mu \mathrm{m}$ ) by a freezing microtome (18). Sections were washed and incubated with blocking buffer (4\% BSA in PBS buffer with Tween-20) for $30 \mathrm{~min}$. It was incubated with anti-TMEM16A (1:200; S-20, no. sc-69343; Santa Cruz Biotechnology, Inc., Dallas, TX, USA) and anti-PAR2 (1:200; ab180953; Abcam, Cambridge, MA, USA) in a wet box overnight at $4^{\circ} \mathrm{C}$. The sections were then washed 4 times with $0.01 \mathrm{M}$ PBS, 5 min each time. Then it was incubated with the solution containing donkey anti-rabbit IgG-FITC (1:200; Santa Cruz Biotechnology, Inc.) and donkey anti-goat IgG-TRITC (1:200; 
Santa Cruz Biotechnology, Inc.) at $37^{\circ} \mathrm{C}$ for $2 \mathrm{~h}$. Slides were then examined by confocal microscopy (LSM710; Carl Zeiss AG, Oberkochen, Germany). Immunofluorescence quantification for PAR2 and TMEM16A expression in the dorsal root ganglion were performed by measuring the mean absorbance following laser confocal microscopy with the use of analysis software (ZEN 2009 Light Edition; Carl Zeiss AG).

Western blot analysis. We harvested the L4-6 DRGs from sham group $(\mathrm{n}=12)$ and days $7(\mathrm{n}=12)$ and $14(\mathrm{n}=12)$ post-CCI. The homogenates were incubated at $4^{\circ} \mathrm{C}$ for $30 \mathrm{~min}$ and centrifuged at $12,000 \mathrm{~g}$ for $40 \mathrm{~min}$ at $4^{\circ} \mathrm{C}$. The supernatant was collected, and the protein concentration in the supernatant was estimated by the BCA protein assay. Each samples with equal amounts of protein $(20 \mu \mathrm{g} / \mathrm{lane})$ were separated by $10 \%$ SDS-PAGE electrophoresis. The resolved proteins were then transferred to a PVDF membrane (EMD Millipore, Billerica, MA, USA). The membranes were blocked with 5\% non-fat milk in TBST buffer (pH 8.0, $10 \mathrm{mmol} / \mathrm{l} \mathrm{Tris-HCl}, 150 \mathrm{mmol} / \mathrm{l} \mathrm{NaCl}$ and $0.2 \%$ Tween 20) for $1 \mathrm{~h}$ at room temperature, and then probed with various primary antibodies [anti-PAR2 antibody (1:250, ab180953; Abcam); anti-TMEM16A antibody (1:1,000, ab53212; Abcam); anti- $\beta$-actin antibody $(1: 5,000$; Merck $\mathrm{KGaA})]$ overnight at $4{ }^{\circ} \mathrm{C}$. After incubation of primary antibodies, the blots were washed three times with TBST, 5 min each time and incubated with secondary antibody [1:10,000; horseradish peroxidase-conjugated goat anti-rabbit or goat anti-mouse secondary antibodies (Alpha Diagnostic, San Antonio, Texas, USA)] for $2 \mathrm{~h}$ at room temperature. The blots were finally washed six times with TBST, 5 min each time and visualized on the X-ray film using the ECL chemiluminescence reagent (GE Healthcare, Chicago, IL, USA). The optical density of each target protein band was assessed with Quantity one software (Bio-Rad Laboratories, Inc., Hercules, CA, USA) and normalized using corresponding $\beta$-actin bands in the same sample (18).

Detection of $I P_{3}$ in dorsal root ganglion by ELISA. We harvested the L4-6 DRGs from sham group $(\mathrm{n}=6)$ and days $7(n=6)$ and $14(n=6)$ post-CCI. The homogenates were centrifuged at 5,000 $\mathrm{g}$ for $5 \mathrm{~min}$. According to the $\mathrm{IP}_{3}$ test kit (CEC037Ge 96T; Cloud-Clone Corp., Wuhan, Hubei, China) instructions, we leave the supernatant to test the concentration of $\mathrm{IP}_{3}(\mathrm{pg} / \mathrm{ml})$ in the dorsal root ganglion of each group (19).

Statistical Analysis. Data were analyzed with SPSS v19.0 software (SPSS, Inc., Chicago, IL, USAs) and presented as the mean \pm standard error of the mean. A homogeneity test for variance was performed followed by one-way analysis of variance with with post hoc Student-Newman-Keuls tests. Two-group comparison was conducted using the least significant difference t-test. $\mathrm{P}<0.05$ was considered to indicate a statistically significant difference.

\section{Results}

Decreased TWL in Hot-plate testing. We first confirmed whether chronic constriction injury of the sciatic nerve cause hyperalgesia or allodynia in rats by withdrawal responses to thermal stimulation. The thermal withdrawal latency from radiant heat was measured at time points of $1,3,5,7,10$ and

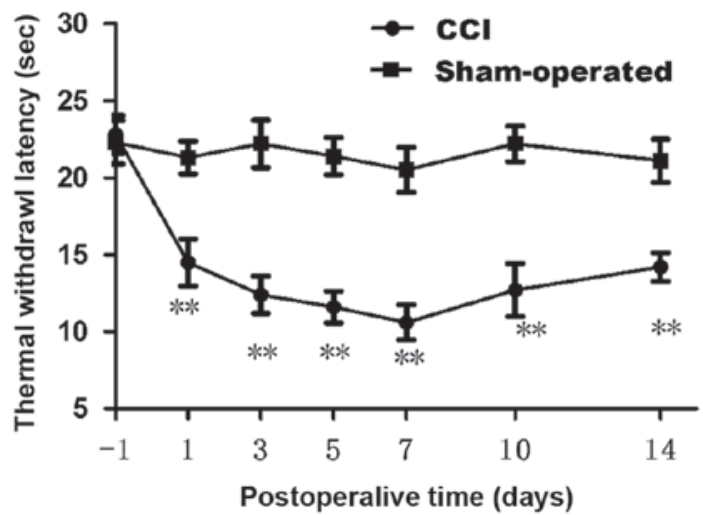

Figure 1. Changes of thermal withdrawal latency (TWL) in sham group and CCI group. Compared with sham group, CCI group presented a dramatic decrease in TWL on 7 day after operation. ${ }^{* *} \mathrm{P}<0.01$ vs. sham group $(n=12)$.

14 day after chronic constriction injury. As shown in Fig. 1, starting from fist day after surgery, apparent dramatically decrease $(n=12 ; P<0.01)$ in the thermal withdrawal latency of ipsilateral paws was observed in CCI model, reflecting a state of persistent hyperalgesia. Comparing with the sham group (21.30 \pm 1.05 at 1 day; $21.10 \pm 1.41$ at 14 day), the TWL of CCI group was decreased significantly from 1 day $(14.50 \pm 1.53)$ to 14 days $(14.20 \pm 0.92)$. Decrease in the thermal pain threshold indicates the success of the CCI model. Low tolerance and paw flinching suggest neuropathic pain in rats. Rats in CCI group presented a dramatic decrease in thermal pain threshold on 7 day after operation. Data are expressed as mean \pm SME $(\mathrm{n}=12 ; \mathrm{P}<0.01 ;$ Fig. 1$)$.

The changes in the expression of PAR2 and TMEM16A after CCI. To confirm effects of neuropathic pain on the PAR2 and TMEM16A protein level, we performed immunoblotting on L4-6 DRGs in sham group, CCI-D7 group and CCI-D14 group. We collected DRG neurons between three groups and analyzed the proteins of PAR2 and TMEM16A. Compared with the sham group, we found that the expression of PAR2 (Fig. 2) and TMEM16A (Fig. 3) protein in the CCI-D7 group and CCI-D14 group increased significantly $(\mathrm{n}=12 ; \mathrm{P}<0.05)$. At the level of protein expression, the relative level of band intensity of PAR2 protein in sham, CCI-D7 and CCI-D14 groups were $0.84,1.02$ and 1.18 respectively (Fig. $2 \mathrm{~B}$ ), the relative level of band intensity of TMEM16A protein in Sham, CCI-D7 and CCI-D14 groups were $0.80,1.02$, and 1.19 respectively (Fig. 3B). In addition, the fluorescence intensity of PAR2 and TMEM16A were increased with the progress of neuropathic pain (Figs. 2C-E; Fig. 3C-E). Furthermore, the changes in protein expression of PAR2 and TMEM16A are consistent with the fluorescence intensity of PAR2 and TMEM16A (Figs. 2, 3). Indeed, these results indicate that CCI induced neuropathic hyperalgesia can up-regulate protein expression of PAR2 and TMEM16A.

Co-expression of PAR2 and TMEM16A proteins on DRG neurons in neuropathic pain rats. To clarify the localization of PAR2 and TMEM16A on DRG neurons, we performed immunofluorescence double staining experiments to identify the expression of the two proteins in DRG neurons. As shown 
$\mathbf{A}$

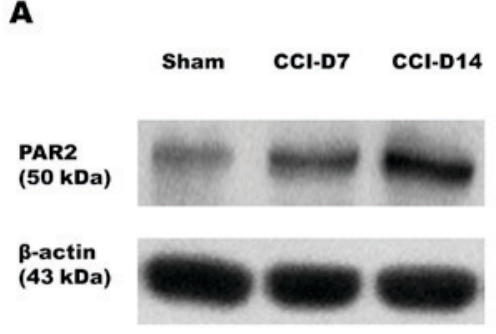

C

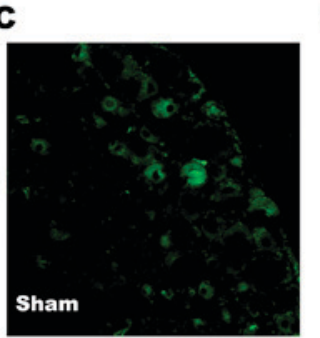

D

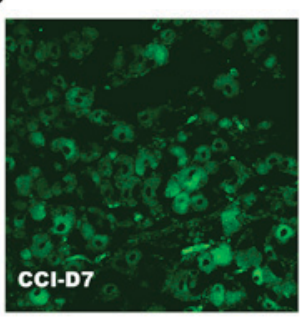

B

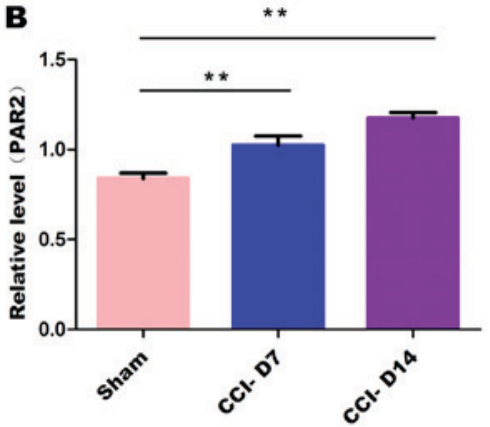

$\mathbf{E}$

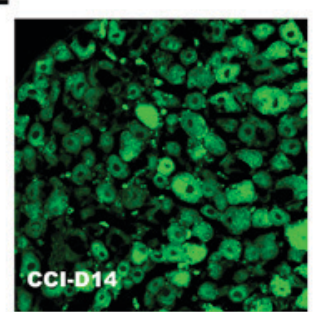

Figure 2. (A) Expression level of PAR2 protein in sham group, CCI-D7 group and CCI-D14 group. (B) Relative level of PAR2 protein expression, data are expressed as mean $\pm \mathrm{SME},{ }^{* *} \mathrm{P}<0.01$ vs. the sham group $(\mathrm{n}=12)$. (C-E) fluorescence intensity of PAR2 in sham, CCI-D7 and CCI-D14 group (n=12), Original magnification, $\mathrm{x} 200$.

A

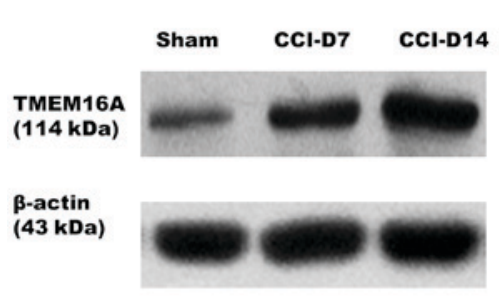

C

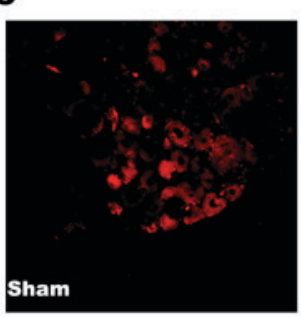

D

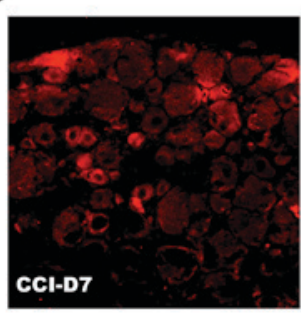

B

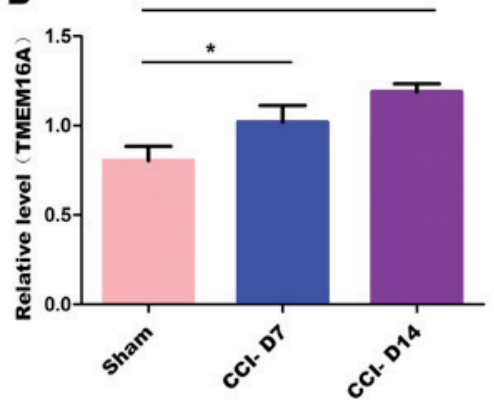

$\mathbf{E}$

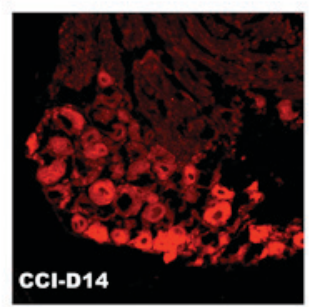

Figure 3. (A) Expression level of TMEM16A protein in sham group, CCI-D7 group and CCI-D14 group. (B) Relative level of TMEM16A protein expression, data are expressed as mean $\pm \mathrm{SME},{ }^{*} \mathrm{P}<0.05$ and ${ }^{* *} \mathrm{P}<0.01$ vs. the sham group $(\mathrm{n}=12)$. (C-E) fluorescence intensity of TMEM16A in sham, CCI-D7 and CCI-D14 groups (n=12), Original magnification, $\mathrm{x} 200$.

in Fig. 4, immunofluorescence double staining experiments showed that PAR2 and TMEM16A were co-expressed in each group of DRG. As shown in Fig. 5, compared with the sham group, the fluorescence intensity of PAR2 in CCI-D7 group was increased $(n=12 ; \mathrm{P}<0.05)$ and in CCI-D14 group was increased significantly $(\mathrm{n}=12 ; \mathrm{P}<0.01)$ (Fig. $5 \mathrm{~A})$; the fluorescence intensity of TMEM16A in CCI-D7 group and CCI-D14 group was increased too $(\mathrm{n}=12 ; \mathrm{P}<0.05)$ (Fig. 5B).

Detection of $\mathrm{IP}_{3}$ concentration in dorsal root ganglion by ELISA. The experimental results by enzyme linked immunosorbent assay shows that compared with the sham group, the concentration of $\mathrm{IP}_{3}$ in CCI-D7 group $(\mathrm{n}=6 ; \mathrm{P}<0.05)$ and CCI-D14 group $(n=6 ; \mathrm{P}<0.01)$ were increased; compared with CCI-D7 group, the concentration of $\mathrm{IP}_{3}$ in CCI-D14 group was increased significantly $(n=6 ; P<0.01)$ (Fig. 6).

\section{Discussion}

Management of neuropathic pain is a major medical issue. In the last decades, great progress have been made in understanding the molecular mechanisms involved in pain generation and perception. Key cellular components for neuropathic pain generation are nociceptors. Nociceptive neural impulses 

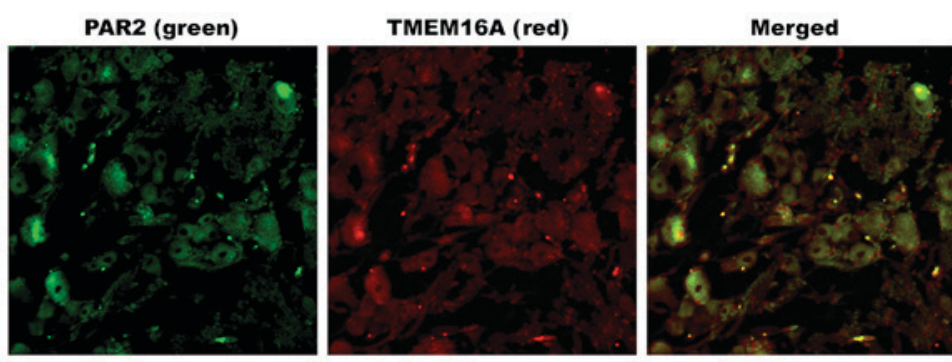

Sham
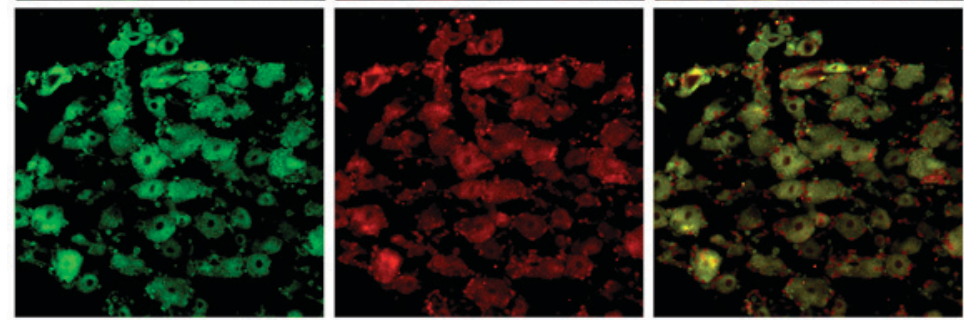

CCI-D7
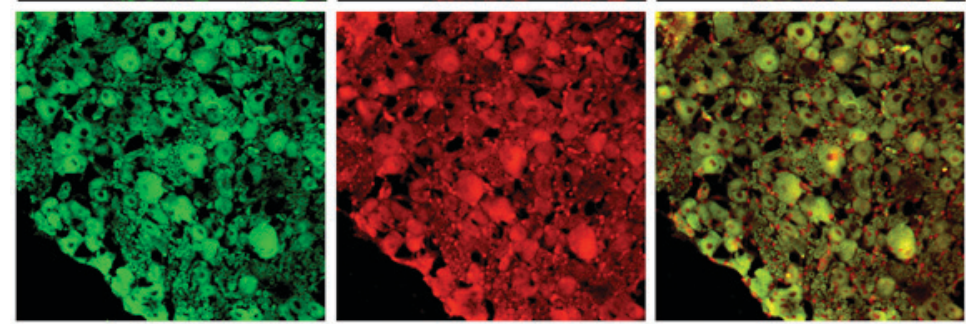

Figure 4. Immunofluorescence co-expression of PAR2 (green) and TMEM16A (red) in sham, CCI-D7 and CCI-D14 groups (n=12). PAR2 and TMEM16A immunoreactivity were seen in DRG neuron cell membranes. Neurites of DRG neurons formed networks. PAR2 positive cells presented green fluorescent labeling, TMEM16A positive cells presented red fluorescent labeling, Mild fluorescence intensity in the sham group, high fluorescence intensity in the CCI-D7 and CCI-D14 group. Original magnification, x200.
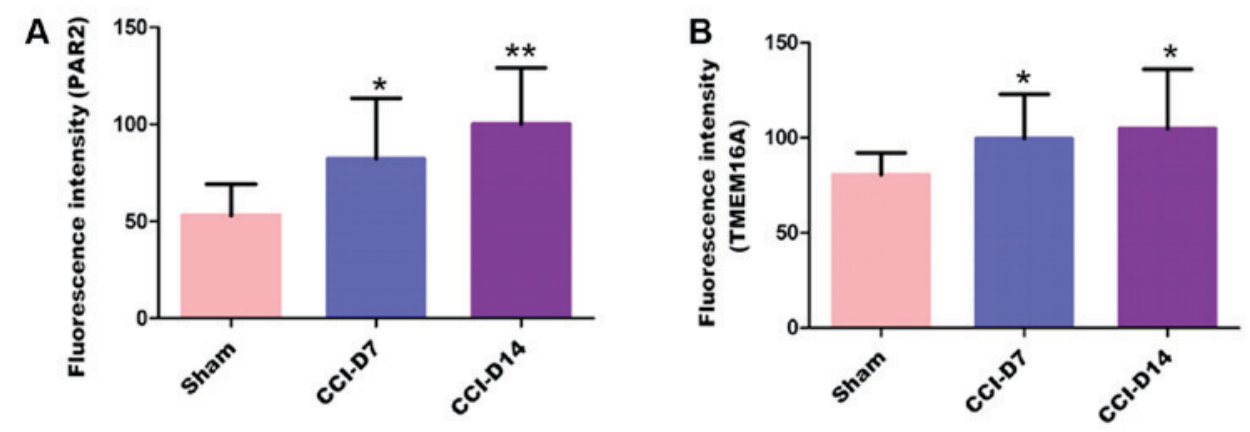

Figure 5. The fluorescence intensity of PAR2 (A) and TMEM6A (B) in sham, CCI-D7 and CCI-D14 groups in dorsal root ganglion neurons. The histogram shows the fluorescence intensity of PAR2 and TMEM6A in the DRG, the fluorescence intensity trend of PAR2 are consistent with the fluorescence intensity trend of TMEM16A. Data are expressed as mean \pm SEM of twelve rats in each group. ${ }^{*} \mathrm{P}<0.05$ and ${ }^{* *} \mathrm{P}<0.01$ vs. the sham group ( $\left.\mathrm{n}=12\right)$.

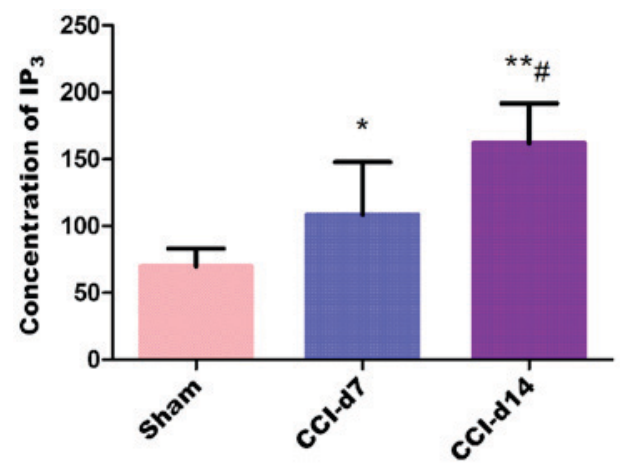

Figure 6. The concentration of $\mathrm{IP}_{3}$ in sham, CCI-D7 and CCI-D14 groups in dorsal root ganglion neurons. The concentration trend of $\mathrm{IP}_{3}$ were consistent with the changes in protein expression of PAR2 and TMEM16A. ${ }^{*} \mathrm{P}<0.05$ and ${ }^{* *} \mathrm{P}<0.01$ vs. the sham group; ${ }^{*} \mathrm{P}<0.05$ vs. the CCI-D7 group $(\mathrm{n}=6)$. originate in primary afferent neurons in dorsal root ganglia, which then activate neurons in the spinal cord and in specific nuclei in the brain to induce the perception of pain. There are many mechanisms for neuropathic pain. The present study showed that some factors expressed in damaged tissues such as histamine, prostaglandins, neuropeptides, cytokines, growth factors, 5-HT, ATP and BK, these substances could increase excitability and result in pain sensitization when the nerve fibers were stimulated (20). Increasing evidence suggests that differential expression of PAR2 and TMEM16A protein results in the development of chronic neuropathic pain induced hyperalgesia. In the present study, we characterized the effects of PAR2 and TMEM16A on the excitability of DRG neurons during CCI induced hyperalgesia. The effects of neuropathic pain on PAR2 and TMEM16A protein expression were evaluated with 
immunocytochemistry and western blot. The aim of our study was to investigate the expression of PAR2 and TMEM16A in the dorsal root ganglion neurons of rats with neuropathic pain model (CCI), and to explore its possible role in neuropathic pain.

$\mathrm{CCI}$, as a novel model of peripheral neuropathic pain, which analogous to the human beings and the symptoms in this rat model. According to Bennett and Xie (21) method, we successfully established animal model of CCI, the main performance is: The left hind limb paw slight adduction deformity and limp, licking the left foot and left hanging leg protective posture. The thermal withdrawal latency, CCI group began to decrease after 1 day, reaching a minimum after 7 days (Fig. 1). The above described is basically the same as Bennett and Xie (21) method.

Previous studies have confirmed that PAR2 were detected in the rat hippocampus, amygdala, thalamus, cortex, hypothalamus, striatum and dorsal root ganglia (22). In our experiment, we found the expression of PAR2 on DRG neurons in the CCI group was gradually increased (Fig. 2A and B), suggesting that PAR2 may be involved in the mechanism of inflammatory or neuropathic pain. Activation of PAR2 may be due to the activation of protein kinase $\mathrm{A}$ and protein kinase $\mathrm{C}$ signaling pathway (23). Activation of PAR2 may increase the neurons excitability which dependented on cGMP activity and cAMP activity and the application of PAR2 inhibitors could inhibit activation of c-AMP and protein kinase A signaling pathway, finally inhibited the hyperalgesia and inflammation (9). During Inflammation, BDNF was also released through the activation of PAR2 signal pathway in microglia (24). At the same time, studies have shown that activation of PAR2 could activate TRPV1, then stimulated the release of neuropeptides SP and CGRP, which can cause the occurrence of hyperalgesia (25-27). In addition, in the PAR2 deficient mice, due to the disappearence of serine protease and depletion of mast cells in mice, pain significantly reduced, so the activation of PAR2 is considered to be the new mechanism of abnormal pain associated with cancer (28), inhibiting the activation of PAR2 may become a new target for the development and treatment of bone cancer pain (29). All of the above studies indicated that PAR2 may be closely related to pain.

Our present study also found that TMEM16A was expressed widely in DRG neurons, and positive cells were evenly distributed in the cytoplasm and cell membrane, the nucleus no mark. In the experimental process, the expression of TMEM16A in the CCI group of DRG neurons was gradually increased compared with the sham group (Fig. 3A and B), suggesting that the expression of TMEM16A is closely related to the progression of neuropathic pain. TMEM16A is not only the molecular basis of calcium activated chloride channels, but also a presence in the nociceptor heat sensitive protein, which mediates many physiological functions. Moreover, hyperalgesia and allodynia were significantly reduced in TMEM16A knockout mice (30). Knockdown of TMEM16A from DRG neurons also attenuated thermal hyperalgesia in both neuropathic pain and inflammatory models (31). In addition, a recent research also suggested that activated pain response could be suppressed by the TMEM16A inhibitor A01 (32). These results suggest that TMEM16A may play a role in neuropathic pain.

Studies have shown that TMEM16A can be activated by an increase in the intracellular $\mathrm{Ca}^{2+}$ concentration through ion channels (33). Recent research have found that TMEM16A can be regulated by mechanical stimuli (34), Protons (35), cholesterol $(36,37)$, calmodulin $(38-40)$ and $\operatorname{IP}_{3}(41,42)$. In our experiment, we found that the concentration of $\mathrm{IP}_{3}$ in dorsal root ganglia was increased following the experimental process (Fig. 6), protein expression of PAR2 and TMEM16A were increased in the same trend, therefore, we assume that increasing expression of two protains may be related to the release of $\mathrm{IP}_{3}$. That is to say, the increasing expression of PAR2 protein caused the increased release of $\mathrm{IP}_{3}$, and the increasing release of $\mathrm{IP}_{3}$ induced the activation of TMEM16A. Recent studies have shown that activation of PAR2 increased calcium ion concentration by release $\mathrm{IP}_{3}(9)$, accompanied by an increase in intracellular calcium concentration, TMEM16A activation threshold reduced and activated current enhanced (14).

In conclusion, these data suggest that PAR2 and TMEM16A were co-expression on DRGs of the primary afferent neurons, which were sensitive to CCI-induced neuropathic pain. CCI-induced the up-regulation expression of PAR2 and TMEM16A may maintain hyperalgesia or allodynia. In addition, this experiment confirmed that expression of PAR2 and TMEM16A were gradually increased with the progression of neuropathic pain duration, and the concentration trend of IP3 were consistent with the changes in protein expression of PAR2 and TMEM16A. But the relationship between PAR2 and TMEM16A in the development of neuropathic pain, and the specific mechanism needs further verification in future experiments.

\section{Acknowledgements}

The authors would like to express their thanks to all those who helped in the writing of this paper. And thanks to the project supported by the National Natural Science Fund (no. 81560175).

\section{References}

1. Jensen TS, Baron R, Haanpää M, Kalso E, Loeser JD, Rice AS and Treede RD: A new definition of neuropathic pain. Pain 152: 2204-2205, 2011.

2. Bowsher D: Neurogenic pain syndromes and their management. Br Med Bull 47: 644-666, 1991.

3. Carter GT and Galer BS: Advances in the management of neuropathic pain. Phys Med Rehabil Clin N Am 12: 447-459, 2001.

4. Toth $\mathrm{C}$, Lander $\mathrm{J}$ and Wiebe S: The prevalence and impact of chronic pain with neuropathic pain symptoms in the general population. Pain Med 10: 918-929, 2009.

5. Mcdermott AM, Toelle TR, Rowbotham DJ, Schaefer CP and Dukes EM: The burden of neuropathic pain: Results from a cross-sectional survey. Eur J Pain 10: 127-135, 2006.

6. O'Connor AB: Neuropathic pain: Quality-of-life impact, costs and cost effectiveness of therapy. Pharmacoeconomics 27: 95-112, 2009.

7. Park GH, Jeon SJ, Ryu JR, Choi MS, Han SH, Yang SI, Ryu JH, Cheong JH, Shin CY and Ko KH: Essential role of mitogen-activated protein kinase pathways in protease activated receptor 2-mediated nitric-oxide production from rat primary astrocytes. Nitric Oxide 21: 110-119, 2009.

8. Bao Y, Hou W and Hua B: Protease-activated receptor 2 signalling pathways: A role in pain processing. Expert Opin Ther Targets 18: 15-27, 2014

9. Huang ZJ, Li HC, Cowan AA, Liu S, Zhang YK and Song XJ: Chronic compression or acute dissociation of dorsal root ganglion induces cAMP-dependent neuronal hyperexcitability through activation of PAR2. Pain 153: 1426-1437, 2012. 
10. Lee B, Cho H, Jung J, Yang YD, Yang DJ and Oh U: Anoctamin 1 contributes to inflammatory and nerve-injury induced hypersensitivity. Mol Pain 10: 5, 2014.

11. García G, Martínezrojas VA, Rochagonzález HI, Granadossoto V and Murbartián J: Evidence for the participation of $\mathrm{Ca}(2+)$-activated chloride channels in formalin-induced acute and chronic nociception. Brain Res 1579: 35-44, 2014.

12. Yang YD, Cho H, Koo JY, Tak MH, Cho Y, Shim WS, Park SP, Lee J, Lee B, Kim BM, et al: TMEM16A confers receptor-activated calcium-dependent chloride conductance. Nature 455 : $1210-1215,2008$

13. Cho H, Yang YD, Lee J, Lee B, Kim T, Jang Y, Back SK, Na HS, Harfe BD, Wang F, et al: The calcium-activated chloride channel anoctamin 1 acts as a heat sensor in nociceptive neurons. Nat Neurosci 15: 1015-1021, 2012.

14. Matsuba S, Niwa S, Muraki K, Kanatsuka S, Nakazono Y, Hatano N, Fujii M,Zhan P, Suzuki T and Ohya S: Downregulation of Ca2+-activated Cl-channel TMEM16A by the inhibition of histone deacetylase in TMEM16A-expressing cancer cells. J Pharmacol Exp Ther 351: 510-518, 2014.

15. Zhao L, Li LI, MA KT, Wang Y, Li J, Shi WY, Zhu HE, Zhang ZS and Si JQ: NSAIDs modulate GABA-activated currents via $\mathrm{Ca} 2+$-activated $\mathrm{Cl}$-channels in rat dorsal root ganglion neurons. Exp Ther Med 11: 1755-1761, 2016.

16. Li L, Zhao L, Wang Y, Ma KT, Shi WY, Wang YZ and Si JQ: PKC $\varepsilon$ mediates substance $\mathrm{P}$ inhibition of GABAA receptors-mediated current in rat dorsal root ganglion. J Huazhong Univ Sci Technolog Med Sci 35: 1-9, 2015.

17. Han YY, Huang Z, Wang YP et al: The study of the expression of potassium sodium chloride transporter on DRG neurons in CC model rat. Chinese Pain Med 22: 583-90, 2016 (In Chinese).

18. Miao XR, Gao XF, Wu JX, Lu ZJ, Huang ZX, Li XQ, He C and Yu WF: Bilateral downregulation of Nav1.8 in dorsal root ganglia of rats with bone cancer pain induced by inoculation with Walker 256 breast tumor cells. BMC Cancer 10: 216, 2010.

19. Yang W, Si-Yuan L, Ke-Tao M, Jun-Qiang S, Lei Z, Zhong-Shuang Z, He Z and Li L: Changes in presynaptic inhibition and the second message system of neuropathic pain model in rats. China J Mod Med 22: 9-14, 2012 (In Chinese).

20. Mcmahon SB, Bennett DLH and Bevan S: Inflammatory mediators and modulators of pain. Churchill Livingstone (Elsevier Health Sciences): 49-72, 2006.

21. Bennett GJ and Xie YK: A peripheral mononeuropathy in rat that produces disorders of pain sensation like those seen in man. Pain 33: 87-107, 1988

22. Striggow F, Riekburchardt M, Kiesel A, Schmidt W, Henrich-Noack P, Breder J, Krug M, Reymann KG and Reiser G: Four different types of protease-activated receptors are widely expressed in the brain and are up-regulated in hippocampus by severe ischemia. Eur J Neurosci 14: 595-608, 2001.

23. Chen Y, Yang C and Wang ZJ: Proteinase-activated receptor 2 sensitizes transient receptor potential vanilloid 1, transient receptor potential vanilloid 4 and transient receptor potential ankyrin 1 in paclitaxel-induced neuropathic pain. Neuroscience 193: 440-451, 2011.

24. Fan Y, Chen J, Ye J, Yan H and Cai Y: Proteinase-activated receptor 2 modulates corticotropin releasing hormone-induced brain-derived neurotrophic factor release from microglial cells. Cell Biol Int 38: 92-96, 2014

25. Spicarova D, Nerandzic V and Palecek J: Update on the role of spinal cord TRPV1 receptors in pain modulation. Physiol Res 63 (Suppl 1): S225-S236, 2014.

26. Spicarova D, Adamek P, Kalynovska N, Mrozkova $P$ and Palecek J: TRPV1 receptor inhibition decreases CCL2-induced hyperalgesia. Neuropharmacology 81: 75-84, 2014.
27. Uchytilova E, Spicarova D and Palecek J: TRPV1 antagonist attenuates postoperative hypersensitivity by central and peripheral mechanisms. Mol Pain 10: 67, 2014.

28. Lam DK, Dang D, Zhang J, Dolan JC and Schmidt BL: Novel animal models of acute and chronic cancer pain: a pivotal role for PAR2. J Neurosci 32: 14178-14183, 2012.

29. Liu S, Liu YP, Yue DM and Liu GJ: Protease-activated receptor 2 in dorsal root ganglion contributes to peripheral sensitization of bone cancer pain. Eur J Pain 18: 326-337, 2014.

30. Stephan AB, Shum EY, Hirsh S, Cygnar KD, Reisert J and Zhao H: ANO2 is the cilial calcium-activated chloride channel that may mediate olfactory amplification. Proc Natl Acad Sci USA 106: 11776-11781, 2009.

31. Liu B, Linley JE, Du X, Zhang X, Ooi L, Zhang H and Gamper N: The acute nociceptive signals induced by bradykinin in rat sensory neurons are mediated by inhibition of M-type $\mathrm{K}+$ channels and activation of $\mathrm{Ca} 2+$-activated $\mathrm{Cl}$-channels. J Clin Invest 120: 1240-1252, 2010.

32. Deba F and Bessac BF: Anoctamin-1 Cl(-) channels in nociception: Activation by an $\mathrm{N}$-aroylaminothiazole and capsaicin and inhibition by T16A (inh)-A01. Mol Pain 11: 55, 2015

33. Ferrera L, Caputo A and Galietta LJ: TMEM16A protein: A new identity for $\mathrm{Ca}(2+)$-dependent $\mathrm{Cl}(-)$ channels. Physiology (Bethesda) 25: 357-363, 2010

34. Bulley S, Neeb ZP, Burris SK, Bannister JP, Thomas-Gatewood CM, Jangsangthong W and Jaggar JH: TMEM16A channels contribute to myogenic constriction in cerebral arteries. Circ Res 111: 1027-1036, 2012.

35. Chun H, Cho H, Choi J, Lee J, Kim SM, Kim H and Oh U: Protons inhibit anoctamin 1 by competing with calcium. Cell Calcium 58: 431-441, 2015.

36. Sones WR, Davis AJ, Leblanc N and Greenwood IA: Cholesterol depletion alters amplitude and pharmacology of vascular calcium-activated chloride channels. Cardiovasc Res 87: 476-484, 2010

37. Cipriani G, Serboiu CS, Gherghiceanu M,Faussone-Pellegrini MS and Vannucchi MG: NK receptors, Substance P, Anol expression and ultrastructural features of the muscle coat in Cav-1(-/-) mouse ileum. J Cell Mol Med 15: 2411-2420, 2011

38. Tian Y, Kongsuphol P, Hug M, Ousingsawat J, Witzgall R, Schreiber R and Kunzelmann K: Calmodulin-dependent activation of the epithelial calcium-dependent chloride channel TMEM16A. FASEB J 25: 1058-1068, 2011.

39. Jung J, Nam JH, Park HW, Oh U, Yoon JH and Lee MG: Dynamic modulation of ANO1/TMEM16A HCO3(-) permeability by Ca2+/calmodulin. Proc Natl Acad Sci USA 110: 360-365, 2013.

40. Vocke K, Dauner K, Hahn A, Ulbrich A, Broecker J, Keller S, Frings S and Möhrlen F: Calmodulin-dependent activation and inactivation of anoctamin calcium-gated chloride channels. J Gen Physiol 142: 381-404, 2013.

41. Tian Y, Schreiber R, Wanitchakool P, Kongsuphol P, Sousa M, Uliyakina I, Palma M, Faria D, Traynor-Kaplan AE, Fragata JI, et al: Control of TMEM16A by INO-4995 and other inositolphosphates. Br J Pharmacol 168: 253-265, 2013.

42. Pritchard HA, Leblanc N, Albert AP and Greenwood IA Inhibitory role of phosphatidylinositol 4,5-bisphosphate on TMEM16A-encoded calcium-activated chloride channels in rat pulmonary artery. Br J Pharmacol 171: 4311-4321, 2014.

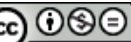

This work is licensed under a Creative Commons Attribution-NonCommercial-NoDerivatives 4.0 International (CC BY-NC-ND 4.0) License. 